\title{
Nodular regenerative hyperplasia of the liver
}

INSERM

\section{Source}

INSERM. (1999). Orphanet: an online rare disease and orphan drug data base. Nodular regenerative hyperplasia of the liver. ORPHA:48372

Nodular regenerative hyperplasia of the liver is a rare parenchymatous liver disease characterized by diffuse benign transformation of the hepatic parenchyma into multiple small nodules (composed of regenerating hepatocytes) and that is usually asymptomatic but can lead to the development of non-cirrhotic portal hypertension and its complications, including esophageal variceal bleeding, hypersplenism and ascites. It is often associated with rheumatologic, autoimmune, hematologic, and myeloproliferative disorders as well as various immune deficiency states and exposure certain drugs and toxins. 